\title{
A reference cytochrome c oxidase subunit I database curated for hierarchical classification of arthropod metabarcoding data
}

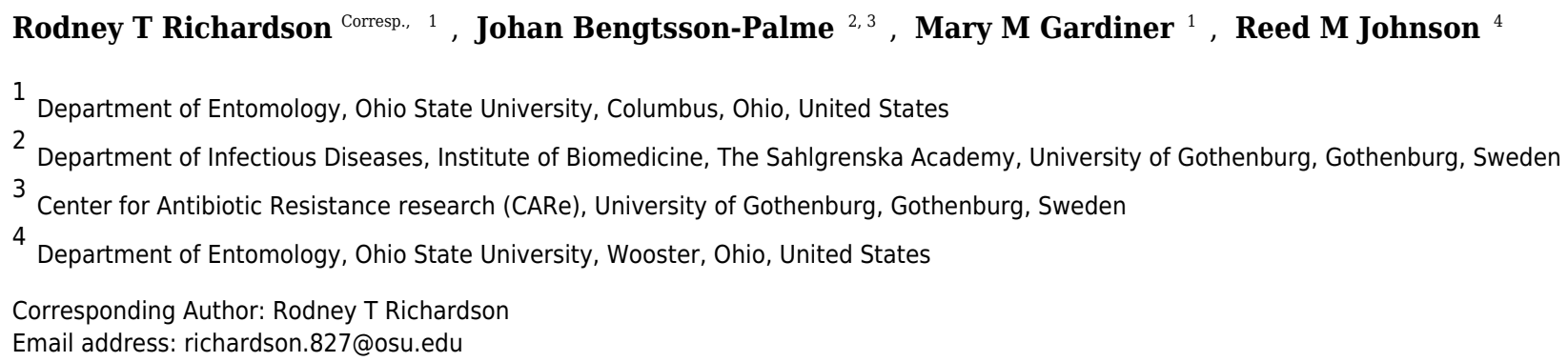

Metabarcoding is a popular application which warrants continued methods optimization. To maximize barcoding inferences, hierarchy-based sequence classification methods are increasingly common. We present methods for the construction and curation of a database designed for hierarchical classification of a $157 \mathrm{bp}$ barcoding region of the arthropod cytochrome c oxidase subunit I (COI) locus. We produced a comprehensive arthropod COI amplicon dataset including annotated arthropod COI sequences and COI sequences extracted from arthropod whole mitochondrion genomes, the latter of which provided the only source of representation for Zoraptera, Callipodida and Holothyrida. The database contains extracted sequences of the target amplicon from all major arthropod clades, including all insect orders, all arthropod classes and Onychophora, Tardigrada and Mollusca outgroups. During curation, we extracted the COI region of interest from approximately 81 percent of the input sequences, corresponding to 73 percent of the genus-level diversity found in the input data. Further, our analysis revealed a high degree of sequence redundancy within the NCBI nucleotide database, with a mean of approximately 11 sequence entries per species in the input data. The curated, lowredundancy database is included in the Metaxa2 sequence classification software ( http://microbiology.se/software/metaxa2/ ). Using this database with the Metaxa2 classifier, we performed a cross-validation analysis to characterize the relationship between the Metaxa2 reliability score, an estimate of classification confidence, and classification error probability. We used this analysis to select a reliability score threshold which minimized error. We then estimated classification sensitivity, false discovery rate and overclassification, the propensity to classify sequences from taxa not represented in the reference database. Our work will help researchers design and evaluate classification databases and conduct metabarcoding on arthropods and alternate taxa. 
1 A Reference Cytochrome C Oxidase Subunit I Database Curated for Hierarchical

2 Classification of Arthropod Metabarcoding Data

3 Rodney T. Richardson ${ }^{1 *}$, Johan Bengtsson-Palme ${ }^{2,3}$, Mary M. Gardiner ${ }^{4}$, Reed M. Johnson ${ }^{1}$

$4{ }^{1}$ Department of Entomology, The Ohio State University-Ohio Agricultural Research and

5 Development Center, 1680 Madison Ave., Wooster, Ohio 44691 USA.

62 Department of Infectious Diseases, Institute of Biomedicine, The Sahlgrenska Academy,

7 University of Gothenburg, Guldhedsgatan 10, SE-413 46, Gothenburg, Sweden

$8{ }^{3}$ Center for Antibiotic Resistance research (CARe) at University of Gothenburg, Box 440, SE-

9 40530, Gothenburg, Sweden

$10{ }^{4}$ Department of Entomology, The Ohio State University, 2021 Coffey Road, Columbus, Ohio

1143210 USA.

12 Corresponding Author:

13 Rodney Richardson

14 Email address: richardson.827@osu.edu 


\section{Abstract}

Metabarcoding is a popular application which warrants continued methods optimization. To maximize barcoding inferences, hierarchy-based sequence classification methods are increasingly common. We present methods for the construction and curation of a database designed for hierarchical classification of a $157 \mathrm{bp}$ barcoding region of the arthropod cytochrome c oxidase subunit I (COI) locus. We produced a comprehensive arthropod COI amplicon dataset including annotated arthropod COI sequences and COI sequences extracted from arthropod whole mitochondrion genomes, the latter of which provided the only source of representation for Zoraptera, Callipodida and Holothyrida. The database contains extracted sequences of the target amplicon from all major arthropod clades, including all insect orders, all arthropod classes and Onychophora, Tardigrada and Mollusca outgroups. During curation, we extracted the COI region of interest from approximately 81 percent of the input sequences, corresponding to 73 percent of the genus-level diversity found in the input data. Further, our analysis revealed a high degree of sequence redundancy within the NCBI nucleotide database, with a mean of approximately 11 sequence entries per species in the input data. The curated, low-redundancy database is included in the Metaxa2 sequence classification software (http://microbiology.se/software/metaxa2/). Using this database with the Metaxa2 classifier, we performed a cross-validation analysis to characterize the relationship between the Metaxa2 reliability score, an estimate of classification confidence, and classification error probability. We used this analysis to select a reliability score threshold which minimized error. We then estimated classification sensitivity, false discovery rate and overclassification, the propensity to classify sequences from taxa not represented in the reference database. Our work will help researchers design and evaluate classification databases and conduct metabarcoding on arthropods and alternate taxa. 


\section{Introduction}

With the increasing availability of high-throughput DNA sequencing, scientists with a wide diversity of backgrounds and interests are increasingly utilizing this technology to achieve a variety of goals. One growing area of interest involves the use of metabarcoding, or amplicon sequencing, for biomonitoring, biodiversity assessment and community composition inference (Yu et al. 2012; Guardiola et al. 2015; Richardson et al. 2015). Using universal primers designed to amplify conserved genomic regions across a broad diversity of taxonomic groups of interest, researchers are afforded the opportunity to survey biological communities at previously unprecedented scales. While such advancements hold great promise for improving our knowledge of the biological world, they also represent new challenges to the scientific community.

Given that bioinformatic methods for taxonomic inference of metabarcoding sequence data are relatively new, the development, validation and refinement of appropriate analytical methods is ongoing. Relatively few studies have characterized the strengths and weaknesses of different bioinformatic sequence classification protocols (Porter et al. 2012; Bengtsson-Palme et al. 2015; Peabody et al. 2015; Somervuo et al. 2016; Richardson et al. 2017). Further, researchers continue to utilize a diversity of methods to draw taxonomic inferences from amplicon sequence data. Relative to alignment-based nearest-neighbor and lowest common ancestor-type classification approaches, methods involving hierarchical classification of DNA sequences are popular as they are often designed to estimate the probabilistic confidence of taxonomic inferences at each taxonomic rank. However, studies explicitly examining the accuracy of classification confidence estimates are rare (Somervuo et al. 2016). 
60

61

62

63

64

When performing hierarchical classification, the construction, curation and uniform taxonomic annotation of the reference sequence database is an important methodological consideration. Database quality can affect classification performance in numerous ways. For example, artifacts within the taxonomic identifiers of a reference database can represent artificial diversity and the inclusion of sequence data adjacent to the exact barcoding locus of interest likely display sequence composition that is unrepresentative of the barcoding locus. Lastly, sequence redundancy within reference databases increases computational resource use and is particularly problematic for classification software programs that classify sequences based on a set number of top alignments. In general, such database artifacts have the potential to bias model selection and confidence estimation both with k-mer style classifiers such as UTAX, SINTAX and the RDP Naïve Bayesian Classifier (Wang et al. 2007; Edgar 2015; Edgar 2016) and alignment-based classification approaches such as Metaxa2 and Megan (Huson et al. 2011; Bengtsson-Palme et al. 2015). Thus, it is important to identify and manage reference sequence database artifacts during curation for optimal downstream classification performance.

The use of molecular barcoding and metabarcoding in arthropod community assessment and gut content analysis has gained popularity in recent years (Corse et al. 2010; Yu et al. 2012; Mollot et al. 2014; Elbrecht and Leese 2017). However, as with other non-microbial taxonomic groups of interest, few researchers have developed hierarchical DNA sequence classification techniques for arthropods (Porter et al. 2014; Tang et al. 2015; Somervuo et al. 2017). Here, we detail the construction, curation and evaluation of a database designed for hierarchical classification of amplicon sequences belonging to a $157 \mathrm{bp}$ COI locus commonly used for arthropod metabarcoding (Zeale et al. 2011). This work will serve as both a resource for those 
82 conducting experiments using arthropod metabarcoding and as a template for future work

83 curating and evaluating hierarchical sequence classification databases.

84 Methods

85

86

87

88

\section{Data collection and curation}

To produce a comprehensive reference set, all COI annotated sequences from Arthropoda as well as three sister phyla, Mollusca, Onychophora and Tardigrada, between 250 and $2500 \mathrm{bp}$ in length were downloaded from the NCBI Nucleotide repository on October $21^{\text {st }}, 2016$ using the search term 'Arthropoda cytochrome oxidase subunit I'. To supplement this collection, all arthropod whole mitochondrion genomes were downloaded from NCBI Nucleotide on March $3^{\text {rd }}$, 2017 using the search term 'Arthropoda mitochondrion genome'. For metagenetic analysis, the inclusion of close outgroup sequences is useful for estimating the sequence space boundaries between arthropods and alternate phyla. The Perl script provided in Sickel et al. (2015) was then used along with the NCBI Taxonomy module (NCBI Resource Coordinators 2018) to retrieve the taxonomic identity of each sequence across each of the major Linnaean ranks, from kingdom to species.

After obtaining the available sequences and rank annotations, we created an intermediate database to obtain extracted barcode amplicons of interest from the reference data using the Metaxa2 database builder tool (v1.0, beta 4; http://microbiology.se/software/metaxa2/). This tool creates the hidden Markov models (HMMs) and BLAST reference databases underpinning the Metaxa2 classification procedures. Prior to extraction, we randomly selected a reference sequence, trimmed it to the exact $157 \mathrm{bp}$ barcode amplicon of interest and designated it as the archetypical reference during database building using the '-r' argument. The section of the arthropod COI gene we trimmed this sequence to is the amplicon product of the commonly used 
105 primers of Zeale et al. (2011). The reference sequence is used in the database builder tool to

106 define the range of the barcoding region of interest, and the software then trims the remainder of

107 the input sequences to this region using the Metaxa2 extractor (Bengtsson-Palme et al. 2015). To

108 increase the accuracy of multiple sequence alignment during this process, we split the original

109 input sequences on the basis of length prior to running the database builder for amplicon

110 extraction, creating four files with sequences of 250-500 bp, 501-600 bp, 601-2500bp and whole

111 mitochondrion genomes. Following sequence extraction, the database builder tool aligns

112 trimmed sequences using MAFFT (Katoh and Standley 2013) and from this alignment the

113 conservation of each residue in the sequence is determined. The most conserved regions are

114 selected for building HMMs using the HMMER package (Eddy 2011). Input sequences that

115 cover most of the barcoding region and are taxonomically annotated are used to build a BLAST

116 (Altschul et al. 1997) database for sequence classification. Finally, the sequences in the BLAST

117 database are aligned using MAFFT, and the intra- and inter-taxonomic sequence identities are

118 calculated to derive meaningful sequence identity cutoffs at each taxonomic level. This entire

119 process is described in more detail in the Metaxa2 2.2 manual

120 (http://microbiology.se/software/metaxa2/) and in Bengtsson-Palme et al. (2018).

After extraction, sequences were then curated by removal of duplicate sequences using

122 the Java code provided with the RDP classifier (v2.11; Wang et al. 2007), which removes

123 identical sequences or any sequence contained within another sequence. At this point, we

124 conducted extensive curation of the available lineage data for the reference sequence database.

125 For references lacking complete annotation at midpoints within the Linnaean lineage, we used

126 Perl regular expression-based substitution to complete the annotation according to established

127 taxonomic authorities, including MilliBase (Sierwald 2017), the Integrated Taxonomic 
128 Information System (http://www.itis.gov) and the phylogenomic analysis of Regier et al. (2010).

129 Table 1 shows the substitutions made. Further, we removed ranks containing annotations

130 reflective of open nomenclature, such as sp., cf. and Incertae sedis, as well as ranks annotated as

131 'undef.' Lastly, we removed entries containing more than two consecutive uncalled base pairs.

132 Upon analyzing the representativeness of this initial database across arthropod classes

133 and insect orders, we found that amplicon sequences from two insect orders, Strepsiptera and

134 Embioptera, were not present in the curated database, likely due to their poor sequence similarity

135 to the reference sequence used to designate the amplicon barcode region of interest. To add

136 Strepsiptera and Embioptera COI amplicons, all NCBI COI sequences belonging to these orders

137 were downloaded on October $10^{\text {th }}, 2017$, curated and added to the Metaxa2 COI database. To

138 improve recovery of amplicons from these insect orders during curation, a representative

139 sequence from both Embioptera and Strepsiptera, representing the $157 \mathrm{bp}$ COI amplicon of

140 interest, was used when building the Metaxa2 database. This retrospective addition of sequences

141 belonging to Strepsiptera and Embioptera contributed 102 and 3 non-redundant reference

142 sequences to the database, respectively. After this final sequence addition step, a Metaxa2

143 database was built to include all curated sequences and this database is available through the

144 Metaxa2 software package (http://microbiology.se/software/metaxa2/).

145 To assess the degree to which our amplicon sequence extraction, dereplication and

146 curation procedures worked, we took inventory of the number of sequences per species in the

147 initial input data as well as the number of sequences and genera present in the data at three points

148 during curation: 1) in the initial input data, 2) following Metaxa2 database builder-based

149 amplicon sequence extraction and 3) in the final database following dereplication and taxonomic 150 curation. 
151

152

153

154

155

156

157

158

159

160

161

162

163

164

165

166

167

168

169

170

171

172

173

\section{Classifier performance evaluation}

For performance evaluations, the methods used were highly similar to those of

Richardson et al. (2017). For three repeated samplings, we randomly selected 10 percent of the curated sequences to obtain testing data, using the remaining 90 percent of sequences to train the Metaxa2 classifier for performance evaluations. To assess the effect of sequence length on classifier performance, we used a Python script to crop the test case sequences to $80 \mathrm{bp}$ in length, approximately half the median length of the original reference sequence dataset. Evaluating classification performance on these short sequences provides a test of the classifiers robustness to sequence length variation and enables estimation of the potential for classifying sequences from short, high-throughput technology, such as 100 cycle single-end Illumina HiSeq sequencing. We then performed the following analyses on both the full-length $(157 \mathrm{bp})$ and halflength $(80 \mathrm{bp})$ test case sequences, separately.

To characterize the relationship between the Metaxa2 reliability score, an estimate of classification confidence, and the probability of classification error, we used the COI trained classifier to classify the testing datasets, requiring the software to classify to the family rank regardless of the reliability score of the assignment. After comparing the known taxonomic identity of each reference test case to the Metaxa2 predicted taxonomic identity, we regressed 5,000 randomly chosen binary classification outcomes, ' 1 ' representing an incorrect classification and ' 0 ' representing a correct classification, against the Metaxa2 reliability score using local polynomial logistic regression in R (v3.3.1; R Core Team 2014) with the span set to a value of 0.5 .

For each of the three testing and training datasets, we classified the testing sequences using Metaxa2 with a reliability score threshold (-R) of 68 . With the resulting classifications, we 
174 compared the known taxonomic identity of each reference test case to its Metaxa2 classification, 175 from kingdom to species, to assess the proportion of true positive, true negative, false negative 176 and false positive predictions. We also calculated false discovery rate, as measured in errors per 177 assignment for each rank.

To assess the rate of taxonomic overclassification at the genus and species levels, we

179

180 searched the testing dataset for sequence cases belonging to arthropod genera and species not represented in the training database. For each of the two ranks, we then determined the case is not represented in the training data, any such classification represents a particular type of misclassification known as an overclassification or overprediction. Lastly, for these sequence cases, we looked at how the classifier performed at the preceding rank (e.g. for the species-level cases, we analyzed classifier performance at the genus-level). Such analysis provides a measure of how well the software is able to perform at the next higher rank for these worst-case-scenario input sequences.

For each order in the testing data, we estimated the family, genus and species-level proportion of sequences assigned and false discovery rate to estimate the degree of variance in performance across major arthropod lineages. For this analysis, the false discovery rate was again defined as the number of errors per assignment. After conducting this analysis, we limited our interpretation of the results to orders with at least 100 tests sequence cases at all of the ranks analyzed, family, genus and species. A Python script which takes the testing sequence taxonomies, training sequence taxonomies and Metaxa2 predicted taxonomies as input and provides the summaries of classification performance described above is provided with the 
196 GitHub repository associated with this work, which is detailed in the Supplemental Information 197 section.

198 Results

199 Following curation and extraction, we obtained 199,206 reference amplicon sequences 200 belonging to 51,416 arthropod species. Over 90 percent of the references were between 142 and $201149 \mathrm{bp}$ in length, with a minimum reference sequence length of $94 \mathrm{bp}$. For the final database 202 creation and classifier training procedure, many reference amplicons were shorter than the 157 203 bp region of interest due to the incompleteness of some reference sequences and the trimming of 204 taxonomically uninformative ends during Metaxa2 training. Prior to this step, 82 percent of the sequences were between 150 and $157 \mathrm{bp}$ in length following the original extraction and these 206 longer sequences can be found at the GitHub repository associated with this work. The taxonomic representativeness of the database across different arthropod classes and insect orders, 208 including the number of families, genera and species in each, are presented in Tables 2 and 3. Analyzing the number of sequences per species in the input reference sequence data, we observed a heavily right-skewed distribution, with a median of 2 and a mean of 11.1 sequences

211 per species (Figure 1A). Further, 32.0 percent of species were represented by 5 or more

212 sequences and 40 species, including Bemisia tabaci and Delia platura, were represented by 213 between 1,000 and 9,736 entries. After conducting amplicon sequence extraction using the 214 Metaxa2 database builder tool, we were able to extract the COI region of interest from 80.8 215 percent of the input sequences, which corresponded to 73.4 percent of the genus-level diversity 216 found in the original input data. Following sequence dereplication, removal of sequences with 217 three or more ambiguous base calls and taxonomic lineage curation, our final database contained 
218 approximately 13 percent of the input extracted sequences, which represented 98.2 percent of the 219 genus-level richness of the input extracted reference amplicon sequences (Figure 1B and 1C) Regressing classification outcome against the Metaxa2 reliability score yielded a similar

221 best fit model for both the $80 \mathrm{bp}$ and full length test sequence datasets (Figure 2). For both

222 regressions, the probability of sequence mis-assignment was below 10 percent for reliability

223 scores above 70. Thus, for our evaluations, we chose a reliability score of 68 , which

224 corresponded to family-level error probabilities of approximately 11.3 percent and 9.5 percent

225 for $80 \mathrm{bp}$ and full length sequences, respectively.

226 In evaluating the performance of our classification database when analyzed with Metaxa2

227 at a reliability score cutoff of 68 , we found consistently low proportions of false positives across

228 all ranks, though the proportion of true positive, true negative and false negatives varied more 229 considerably from kingdom to species (Figure 3A). Further, while the Metaxa2 false discovery 230 rate increased with higher resolution ranks (Figure 3B), it was generally low, never exceeding 5 231 percent at the genus level. Interestingly, Metaxa2 displayed low variance in the proportion of 232 sequences assigned when classifying $80 \mathrm{bp}$ sequences relative to full length sequences of $147 \mathrm{bp}$ 233 in median length. Overall, the proportion of sequences assigned was greater than 90 percent 234 through the order level for both full length and half length sequences. Beyond the order level, 235 this statistic decreased to 53 and 56 percent at the species level for half length and full length 236 sequences, respectively. Conversely, the proportion of false positives varied more strongly by 237 sequence length and was greatest at higher-resolution taxonomic levels. At the species level, 1.97 percent of $80 \mathrm{bp}$ sequences were misclassified, compared to only 1.13 percent for full length sequences. At the order level, the percent of sequences misclassified was 0.59 and 0.65 for $80 \mathrm{bp}$ and full length sequences, respectively. As measured in errors per assignment, the classification 
241 false discovery rate was similarly highest at the species level, with 7.3 and 6.3 percent of

242 assignments being incorrect for $80 \mathrm{bp}$ and full length sequences, respectively. False discovery

243 rates decreased to 1.2 and 0.7 percent of assignments being incorrect at the order level for $80 \mathrm{bp}$

244 and full length sequences, respectively.

245 During our evaluation of taxonomic overclassifiction, we found between 3,141 and 3,202

246 sequence test cases belonging to species not represented in the corresponding training data and

247 between 612 and 630 sequence test cases belonging to genera not represented in the

248 corresponding training data across the three iterations of training and testing data. At the species

249 level, the proportion of these cases which were overclassified was roughly equal for full length

250 and half length sequences, with 5.4 and 5.2 percent being overclassified (Figure 4A). With

251 respect to genus level classification performance on these species overclassification test cases,

252 approximately 31 and 37 percent of test cases were classified correctly as true positives or true

253 negatives for half length and full length sequences. Genus level false positive proportions for

254 these sequence cases were 6.2 and 5.1 percent (Figure 4B). For the genus level overclassification

255 cases, the difference in overclassification rates by sequence length was slightly larger, with

256 approximately 8.9 and 10.9 percent of full length and half length test cases being overclassified

257 (Figure 4C). Family level performance on genus level overclassification cases was slightly lower

258 with the proportion of true positive and true negative identifications summing to 25 and 27

259 percent for half length and full length sequences, with corresponding false positive proportions

260 of 8.8 and 8.0

261 Evaluating classification performance for each order resulted in unsurprising outcomes

262 across taxonomic ranks, from family to species. Generally, the proportion of sequences assigned

263 decreased and false discovery rate increased with increasing taxonomic resolution. Between 
264 orders, there was noteworthy variation in performance. For example, the proportion of sequences

265 assigned was highest among trichopteran sequences and lowest among lepidopteran sequences,

266 with approximately 94 and 50 percent of sequences being assigned to the family rank for each

267 order, respectively. Further, while the proportion of sequences assigned was similarly lowest for

268 lepidopteran sequences at the genus and species levels, wherein 39 and 21 percent of sequences

269 were assigned for each respective rank, the highest proportion of sequences assigned at these

270 ranks was not observed with trichopteran sequences. Instead, genus and species level proportion

271 assigned was highest among Sessilia sequences, with 86 and 78 percent of sequences being

272 assigned to each respective rank.

\section{Discussion}

274 While species-specific PCR and immunohistochemistry-based methods have been useful

275 in documenting arthropod food webs (Stuart and Greenstone, 1990; Symondson 2002; Weber et

276 al. 2006; Blubaugh et al. 2016), the narrow species-by-species nature of such approaches has

277 limited their utility for answering large-scale or open-ended ecological questions. With the

278 increasing availability of high-throughput sequencing, arthropod metabarcoding will continue to

279 become more broadly applicable to scientific questions spanning a diversity of research areas.

280 The development of improved methods for drawing maximal inferences from sequence data is an

281 important area for further methodological research. In creating a highly curated COI reference

282 amplicon sequence database and evaluating its performance when used with the Metaxa2

283 taxonomic classifier, we have developed a new method to aid researchers in the analysis of

284 arthropod metabarcoding data.

Though predictions vary greatly, researchers have estimated the species richness of

arthropods to be between 2.5 to 3.7 million (Hamilton et al. 2010). Further, according to the 
287 literature review of Porter et al. (2014), 72,618 insect genera have been described to date. Thus

288 the 51,416 species and 17,039 genera represented in our database account for only a small

289 fraction of arthropod biodiversity. The limited representativeness of currently available, high

290 quality reference sequence amplicons for the COI region highlights the need for continued

291 efforts to catalogue arthropod biodiversity with molecular techniques. Despite this current

292 limitation, the combination of molecular gut content analysis with high-throughput sequencing is

293 a promising path toward investigating arthropod trophic ecology and biodiversity monitoring

294 with greater sensitivity and accuracy relative to alternate approaches.

The results of our inventory of sequences per species and genus level richness at various

296

297

298

299

300

301

302

303

304

305

306

307

308

309 stages in the database curation process revealed that our amplicon extraction procedure was

highly sensitive, extracting and trimming approximately 81 percent of the input sequences down

represented sequence redundancies and were removed during dereplication. As mentioned

previously, the trimming of sequence residues adjacent to the barcode of interest and removal of redundant sequences not only makes computational analysis less resource intensive, it can also improve classification performance. For k-mer style classifiers, extraneous sequence residues can bias model selection during classifier training, while abundant sequence duplicates can result in an overwhelming number of identical top hit alignments for alignment-based classifiers.

Overall, the best fit local regression models summarizing the relationship between the Metaxa2 reliability score and the probability of classification error were useful in that the likelihood of misclassification was always less than what would be expected based on the reliability score. For example, a reliability score of 90 corresponded to only a 3.3 percent probability of family-level misclassification for full length sequences. We selected a reliability 
310 score of 68 for subsequent analysis as this provided a balanced trade-off between sensitivity and

311 accuracy. Using this reliability score we observed minimal false positive rates and overall

312 proportions of misclassification when comparing our results to those of similar studies (Porter et

313 al. 2014; Bengtsson-Palme et al. 2015; Edgar 2016; Richardson et al. 2017). Given that the

314 family level probability of error was only 9.5 percent at a reliability score of 68 , a lower

315 reliability score threshold may be justifiable for certain research situations. However, further

316 testing should be conducted to ensure that the relationship between reliability score and

317 classification confidence is similar across taxonomic ranks and between different DNA barcodes.

318 With respect to sensitivity using a reliability score of 68 , our results were highly

319 dependent upon the rank being analyzed, with sensitivities, as measured by the total proportion

320 of sequences classified, above 60 percent only being achieved at the family and order ranks. To

321 some degree, these sensitivity estimates reflect the large degree of database incompleteness at the

322 genus and species ranks, wherein approximately 3.6 and 25.5 percent of unclassified sequences

323 were true negatives. However, to our knowledge, no other studies have reported classification

324 sensitivity data for this COI amplicon locus. This makes it difficult to ascertain if Metaxa2 -

325 with an average of approximately 44.7 percent of false negative assignments at the genus and

326 species ranks - exhibits relatively low sensitivity or if this locus is limited in discriminatory

327 power. The short length of the $157 \mathrm{bp}$ COI amplicon region relative to other barcoding regions

328 such as the ITS and 18S rRNA regions (Hugerth et al. 2014; Wang et al. 2015) could be a cause

329 of such limited discriminatory power.

330 As expected, analyzing cases of overclassification in our data revealed that sequences

331 from taxa lacking representation in the database are far more likely to be misclassified relative to

332 sequences from well-represented taxa. This is supported by an approximately 10 percent 
333 probability of genus level overclassification for sequences from unrepresented genera relative to

334 a 1 to 2 percent probability, depending on sequence length, for all sequence test cases.

335 Interestingly, the genus level overclassification rate was approximately double that observed at

336 the species level. This seems counterintuitive but is expected in light of the discussion put forth

337 by Edgar (2018), wherein the percent identity difference from the closest reference sequence

338 match is considered one of the most important predictors of classification performance. For a

339 species-level overclassification case, the closest reference sequence match in the corresponding

340 database is a sequence from a congeneric species in the best case scenario. Since the closest

341 reference sequence match for a genus level overclassification case is a sequence from a

342 confamilial species at best, the average percent identity difference from the closest reference is

343 greater for a genus level overclassification case than for a species level overclassification case.

344 Thus, for overclassification cases in particular, higher levels of error are expected at more

345 inclusive taxonomic ranks.

346 While the genus level overclassification estimates we observed are not desirable, they are

347 considerably lower than similar estimates for the RDP classifier, which range from 21.3 percent

348 to 67.8 percent depending on the database, locus analyzed and cross-validation approach used

349 (Edgar 2016; Richardson et al. 2017). Further, the observed degree of genus level

350 overclassification using Metaxa2 with our COI database was similar to or less than that of the

351 recently developed SINTAX classifier (Edgar 2016, Edgar 2018). Interestingly, the recent

352 analysis of Edgar (2018), resulted in a similar estimate of the Metaxa2 genus level

353 overclassification rate while revealing a considerably higher overclassification rate for the

354 majority of other classifiers tested. Though this analysis found Metaxa2 to be relatively less

355 sensitive than other classifiers, a weakness of the work was that it only tested Metaxa2 using the 
356 default reliability score of 80 , while testing multiple confidence thresholds for alternate

357 classifiers, such as SINTAX and RDP. Given that our analysis has revealed the Metaxa2 default

358 reliability score to be too conservative - at least for the COI locus - such results are difficult to

359 interpret. In general, such comparisons across studies should be approached with caution as

360 multiple factors complicate the interpretation of classification performance, such as locus

361 discriminatory power, database completeness and the choice of evaluation metrics used.

362 Ultimately, direct comparisons of classification methods using standardized loci and databases

363 are needed to more rigorously compare performance.

364 With respect to Metaxa2 classification of full length relative to half length amplicon

365 sequences, we observed surprisingly small differences in performance. Consistently, the

366 proportion of misclassified sequences was greater for half length sequences. When considering

367 error and sensitivity together, the false discovery rate or errors per assignment for full length

368 sequences was consistently less than or equal to that achieved during the classification of half

369 length sequences. Lastly, when considering the relationship between the Metaxa2 reliability

370 score and the probability of classification error at the family level, we noted highly similar local

371 polynomial regression models of error probability for both full length and half length sequences.

\section{Conclusions}

Here, we assembled a highly curated database of arthropod COI reference amplicon and conducted extensive in silico performance evaluations on the resulting classification pipeline. Overall, we found a high degree of sequence redundancy within the initial, uncurated

377 dataset, highlighting the importance of effective sequence dereplication during the creation of

378 databases designed for metabarcoding analysis. Further, the limited representativeness of the 
379 database with respect to arthropod biodiversity indicates that additional sequencing effort is

380 needed to further improve the performance of arthropod metabarcoding techniques. Though the

381 performance evaluations presented in this work were conducted on a large corpus of available

382 biological data, the results are not necessarily directly transferable to all experimental settings.

383 For example, variations in sequence error profiles and taxonomic distributions among datasets

384 are potential confounding factors. Despite these limitations, this work provides researchers with

385 a new resource for arthropod COI sequence analysis and novel data for gauging the strengths and

386 limitations of different approaches to arthropod metabarcoding.

387 Acknowledgments

388 This work was supported by an allocation of computing time from the Ohio Supercomputer

389 Center.

390 Data Availability

391 The entire set of curated sequences associated with this work can be downloaded from the 392 associated GitHub repository: https://github.com/RTRichar/Zeale_COI_Database. This

393 repository has additionally been archived using Zenodo (DOI: 10.5281/zenodo.1256869)

\section{References}

395 Altschul SF, Madden TL, Schäffer AA, Zhang J, Zhang Z, Miller W, Lipman DJ (1997) Gapped

396 BLAST and PSI-BLAST: a new generation of protein database search programs. Nucleic 397 Acids Research, 25, 3389-3402.

398 Bazinet AL, Cummings MP (2012) A comparative evaluation of sequence classification 399 programs. BMC Bioinformatics, 13, 1-13. 
400 Bengtsson-Palme J, Hartmann M, Eriksson KM, Pal C, Thorell K, Larsson DGJ, Nilsson RH

401

402

403

404

405

406

407

408

409

410

411

412

413

414

415

416

417

418

419

420

421

(2015) Metaxa2: Improved identification and taxonomic classification of small and large subunit rRNA in metagenomic data. Molecular Ecology Resources, 15, 1403-1414.

Bengtsson-Palme J, Richardson RT, Meola M, Wurzbacher C, Tremblay ED, Thorell K, Kanger K, Eriksson M, Bilodeau GJ, Johnson RM, Hartmann M, Nilsson RH (2018) Taxonomic identification from metagenomic and metabarcoding data using any genetic marker. bioRxiv, 253377, doi: https://doi.org/10.1101/253377

Benson DA, Karsch-Mizrachi I, Lipman DJ, Ostell J, Wheeler DL (2005) GenBank. Nucleic Acids Research, 33, D34-D38.

Blubaugh CK, Hagler JR, Machtley SA, Kaplan I (2016) Cover crops increase foraging activity of omnivorous predators in seed patches and facilitate weed biological control. Agriculture, Ecosystems and Environment, 231, 264-270.

Corse E, Costedoat C, Chappaz R, Pech N, Martin J-F, Gilles A (2010) A PCR-based method for diet analysis in freshwater organisms using $18 \mathrm{~S}$ rDNA barcoding on faeces. Molecular Ecology Resources, 10, 96-108.

Eddy SR (2011) Accelerated profile HMM searches. PLoS Computational Biology, 7, e1002195. Edgar RC (2015) UTAX algorithm. Available at: http://www.drive5.com/usearch/manual/utax_algo.html (accessed 6 January 2016).

Edgar RC (2016) SINTAX: A simple non-Bayesian taxonomy classifier for 16S and ITS sequences. Biorxiv. https://doi.org/10.1101/074161.

Edgar RC (2018) Accuracy of taxonomy prediction for 16S rRNA and fungal ITS sequences. PeerJ, 6, e4652. 
422 Elbrecht V and Leese F (2017) Validation and development of COI metabarcoding primers for 423 freshwater macroinvertebrate bioassessment. Frontiers in Environmental Science, 5, 11.

424 Guardiola M, Uriz MJ, Taberlet P, Coissac E, Wangensteen OW, Turon X (2015) Deep-sea, 425 deep-sequencing: Metabarcoding extracellular DNA from sediments of marine canyons. PloS One, 10, e0139633.

427

428

Hamilton AJ, Basset Y, Benke KK, Grimbacher PS, Miller SE, Novotny V, Samuelson A, Stork NE, Weiblen GD, Yen JDL (2010) Quantifying uncertainty in estimation of tropical arthropod species richness. The American Naturalist, 175, 90-95.

Hugerth LW, Muller EEL, Hu YOO, Lebrun LAM, Roume H, Lundin D, Wilmes P, Andersson AF (2014) Systematic design of 18S rRNA gene primers for determining eukaryotic diversity in microbial consortia. PloS ONE, 9, e95567.

Huson DH, Mitra S, Ruscheweyh H-J, Weber N, Schuster SC (2011) Integrative analysis of environmental sequences using MEGAN 4. Genome Research, 21, 1552-1560.

Katoh K, Standley DM (2013) MAFFT multiple sequence alignment software version 7: improvements in performance and usability. Molecular Biology and Evolution, 30, 772780.

Keller A, Schleicher T, Schultz J, Müller T, Dandekar T, Wolf M (2009) 5.8S-28S rRNA interaction and HMM-based ITS2 annotation. Gene, 430, 50-57.

Mollot G, Duyck P-F, Lefeuvre P, Lescourret F, Martin J-F, Piry S, Canard A, Tixier P (2014) Cover cropping alters the diet of arthropods in a banana plantation: A metabarcoding approach. PloS ONE, 9, e93740.

NCBI Resource Coordinators (2018) Database Resources of the National Center for Biotechnology Information. Nucleic Acids Research, 46, D8-D13. 
445 Ohio Supercomputer Center (1987) Citation. Columbus, Ohio, USA.

446 http://osc.edu/ark:/19495/f5s 1 ph73.

447 Peabody MA, Van Rossum T, Lo R, Brinkman FSL (2015) Evaluation of shotgun metagenomics

448 sequence classification methods using in silico and in vitro simulated communities. BMC

$449 \quad$ Bioinformatics, 16, 363.

450 Porter TM, Golding GB (2012) Factors that affect large subunit ribosomal DNA amplicon

451 sequencing studies of fungal communities: Classification method, primer choice, and

$452 \quad$ error. PloS One, 7, e35749.

453 Porter TM, Gibson JF, Shokralla S, Baird DJ, Golding GB, Hajibabaei M (2014) Rapid and accurate taxonomic classification of insect (class Insecta) cytochrome c oxidase subunit 1 (COI) DNA barcode sequences using a naive Bayesian classifier. Molecular Ecology Resources 14, 929-942.

457

R Core Team (2014) R: A language and environment for statistical computing. Vienna, Austria. http://www.R-project.org/

Regier JC, Shultz JW, Zwick A, Hussey A, Ball B, Wetzer R, Martin JW, Cunningham CW (2010) Arthropod relationships revealed by phylogenomic analysis of nuclear protein-

Richardson RT, Bengtsson-Palme J, Johnson RM (2017) Evaluating and optimizing the coding sequences. Nature, 463, 1079-1083. performance of software commonly used for the taxonomic classification of DNA metabarcoding sequence data. Molecular Ecology Resources 17, 760-769. characterization of pollen assemblages collected by honey bees using a multi-locus metabarcoding approach. Applications in Plant Sciences, 3, 1500043. 
468 Sickel W, Ankenbrand MJ, Grimmer G, Holzschuh A, Härtel S, Lanzen J, Steffan-Dewenter I, 469 Keller A (2015) Increased efficiency in identifying mixed pollen samples by meta-

470 barcoding with a dual-indexing approach. BMC Ecology, 15, 1-9.

471 Sierwald P (2017) MilliBase. Accessed at http://www.millibase.org on 2017-04-12

472 Somervuo P, Koskela S, Pennanen J, Nilsson RH, Ovaskainen O (2016) Unbiased probabilistic taxonomic classification for DNA barcoding. Bioinformatics, 32, 2920-2927.

474 Somervuo P, Yu DW, Xu CCY, Ji Y, Hultman J, Wirta H, Ovaskainen O (2017) Quantifying uncertainty of taxonomic placement in DNA barcoding and metabarcoding. Methods in Ecology and Evolution, 8, 398-407.

477

478

479

480

481

482

483

484

485

486

487

488 for identification of predator stomach contents. Annals of the Entomological Society of America, 83, 1101-1107.

Symondson WOC (2002) Molecular identification of prey in predator diets. Molecular Ecology, 11, 627-641.

Tang M, Hardman CJ, Ji Y, Meng G, Liu S, Tan M, Yang S, Moss ED, Wang J, Yang C, Bruce C, Nevard T, Potts SG, Zhou X, Yu DW (2015) High-throughput monitoring of wild bee diversity and abundance via mitogenomics. Methods in Ecology and Evolution, 6, 10341043.

Wang Q, Garrity GM, Tiedje JM, Cole JR (2007) Naïve Bayesian classifier for rapid assignment of rRNA sequences into the new bacterial taxonomy. Applied and Environmental Microbiology, 73, 5261-5267. 
489 Wang X-C, Liu C, Huang L, Bengtsson-Palme J, Chen H, Zhang J-H, Cai D, Li J-Q (2015)

490

491

492

493

494

495

496

497

498

499

500

501

502

503

504

505

506

507

508

509

510

511

ITS1: A DNA barcode better than ITS2 in eukaryotes? Molecular Ecology Resources, 15, 573-586.

Weber DC, Rowley DL, Greenstone MH, Athanas MM (2006) Prey preference and host suitability of the predatory and parasitoid carabid beetle, Lebia grandis, for several species of Leptinotarsa beetles. Journal of Insect Science, 6, 1-14.

Yu DW, Ji Y, Emerson BC, Wang X, Ye C, Yang C, Ding Z (2012) Biodiversity soup: Metabarcoding of arthropods for rapid biodiversity assessment and biomonitoring. Methods in Ecology and Evolution, 3, 613-623.

Zeale MRK, Butlin RK, Barker GLA, Lees DC, Jones G (2011) Taxon-specific PCR for DNA barcoding arthropod prey in bat faeces. Molecular Ecology Resources, 11, 236-244.

\section{Tables}

Table 1: Summary of taxonomic annotations made for references which had undefined ranks at midpoints in their respective taxonomic lineages.

Table 2: Summary of taxonomic representation across all arthropod classes and associated sister groups. Numbers may include sub and super groupings.

Table 3: Summary of insect taxa included in the arthropod COI database following curation.

Numbers may include sub and super groupings.

\section{Figures}

Figure 1: A percent density histogram of the number of sequences per species (A) shows the distribution of redundancy within the NCBI Nucleotide entries used. The dashed blue line and solid red line indicate the median and mean number of sequences per species, respectively.

Inventories of the number of sequences (A) and genera (B) input into the curation process, 
512 following Metaxa2 extraction and following dereplication of redundant sequences and curation

513 of taxonomic lineages.

514 Figure 2: A logistic regression analysis of case-by-case classification accuracy, '1' indicating a

515 false-positive identification and ' 0 ' indicating a true-positive identification, regressed against

516 classification reliability score for half length (A) and full length (B) test sequence cases. A best

517 fit local polynomial regression line (solid blue with 95 percent confidence interval) was used to

518 estimate the relationship between reliability score and the probability of mis-classification.

519 Dashed red lines illustrate the hypothetically ideal 1 to 1 relationship between error probability

520 and the Metaxa2 reliability score, an estimate of classification confidence. Solid black lines

521 highlight the 10 percent error probability.

522 Figure 3: Mean proportion and standard error of true positives (TP), true negatives (TN), false

523 negatives (FN) and false positives (FP) for the classification of all testing sequences, conducted

524 on both the full-length and half-length sequences (A). Mean and standard error of the false

525 discovery rate for both full length and half length sequences, as measured in errors per

526 assignment, during classification of all testing sequences (B).

527 Figure 4: Proportional species level overclassification rate (A) and genus level classification

528 performance (B) for test sequence cases from species not represented in the corresponding

529 training data. Proportional genus level overclassification rate (C), and family level classification

530 performance (D) for test sequence cases from genera not represented in the corresponding

531 training data.

532 Figure 5: Mean and standard error of the proportion of sequences assigned and false discovery

533 rate, as measured in errors per assignment, measured at the family, genus and species levels for

534 sequences belonging to each order. These results represent classification performance by 
535 arthropod order for the full length sequences only and orders with fewer than 100 test sequence 536 cases at any of the ranks analyzed were excluded from analysis. 


\section{Table $\mathbf{1}$ (on next page)}

Taxonomic midpoint annotation corrections.

Summary of taxonomic annotations made for references which had undefined ranks at midpoints in their respective taxonomic lineages. 


\begin{tabular}{|c|c|c|c|}
\hline $\begin{array}{l}\text { Undefined } \\
\text { Rank }\end{array}$ & Higher Resolution Assignment & Assignment Made & Authority Used \\
\hline Order & Family Sphaerotheriidae & Order Sphaerotheriida & \multirow[t]{2}{*}{ MilliBase } \\
\hline Order & Family Zephroniidae & Order Sphaerotheriida & \\
\hline Order & Family Lepidotrichidae & Order Zygentoma & \multirow[t]{10}{*}{ ITIS } \\
\hline Order & Family Lepismatidae & Order Zygentoma & \\
\hline Order & Family Nicoletiidae & Order Zygentoma & \\
\hline Class & Order Pauropoda & Class Myriapoda & \\
\hline Genus & Genus Pseudocellus & Family Ricinididae & \\
\hline Genus & Genus Chanbria & Family Eremobatidae & \\
\hline Genus & Species Tanypodinae spp. & Genus Tanypodinae & \\
\hline Genus & Species Ennominae spp. & Genus Ennominae & \\
\hline Genus & Genus Dichelesthiidae & Family Dichelesthiidae & \\
\hline Genus & Genus Phallocryptus & Family Thamnocephalidae & \\
\hline Class & Order Symphyla & Class Myriapoda & \multirow[t]{5}{*}{ Regier et al. 2010} \\
\hline Order & Family Peripatidae & Order Onychophora & \\
\hline Class & Family Peripatidae & Class Onychophora & \\
\hline Order & Family Peripatopsidae & Order Onychophora & \\
\hline Class & Family Peripatopsidae & Class Onychophora & \\
\hline Genus & Genus Lasionectes & Family Speleonectidae & \multirow[t]{5}{*}{ WoRMS } \\
\hline Family & Family Speleonectidae & Order Nectiopoda & \\
\hline Genus & Genus Prionodiaptomus & Family Diaptomidae & \\
\hline Family & Family Diaptomidae & Order Calanoida & \\
\hline Order & Order Calanoida & Class Maxillopoda & \\
\hline
\end{tabular}




\section{Table 2 (on next page)}

Summary of database completeness with respect to all arthropods.

Summary of taxonomic representation across all arthropod classes and associated sister groups. Numbers may include sub and super groupings. 


\begin{tabular}{l|l|l|l|l}
\hline Class & $\begin{array}{l}\text { Number of } \\
\text { Orders }\end{array}$ & $\begin{array}{l}\text { Number of } \\
\text { Families }\end{array}$ & $\begin{array}{l}\text { Number of } \\
\text { Genera }\end{array}$ & $\begin{array}{l}\text { Number of } \\
\text { Species }\end{array}$ \\
\hline Heterotardigrada & 1 & 2 & 2 & 1 \\
\hline Eutardigrada & 1 & 3 & 12 & 20 \\
\hline Onychophora & 1 & 2 & 17 & 42 \\
\hline Pycnogonida & 1 & 10 & 27 & 89 \\
\hline Cephalopoda & 1 & 1 & 1 & 1 \\
\hline Merostomata & 1 & 1 & 3 & 4 \\
\hline Arachnida & 17 & 226 & 740 & 1804 \\
\hline Myriapoda & 2 & 4 & 7 & 9 \\
\hline Chilopoda & 5 & 16 & 53 & 172 \\
\hline Diplopoda & 11 & 33 & 95 & 181 \\
\hline Ostracoda & 2 & 6 & 19 & 40 \\
\hline Branchiopoda & 3 & 25 & 76 & 254 \\
\hline Malacostraca & 13 & 256 & 969 & 2654 \\
\hline Maxillopoda & 11 & 85 & 240 & 568 \\
\hline Cephalocarida & 1 & 1 & 2 & 1 \\
\hline Remipedia & 1 & 2 & 5 & 8 \\
\hline Protura & 1 & 4 & 12 & 13 \\
\hline Diplura & 1 & 5 & 7 & 11 \\
\hline Collembola & 4 & 18 & 98 & 203 \\
\hline Insecta & 29 & 789 & 14654 & 45341 \\
\hline Total & $\mathbf{1 0 7}$ & $\mathbf{1 4 8 9}$ & $\mathbf{1 7 0 3 9}$ & $\mathbf{5 1 4 1 6}$ \\
\hline
\end{tabular}

1 


\section{Table 3(on next page)}

Summary of database completeness with respect to insects.

Summary of insect taxa included in the arthropod COI database following curation. Numbers may include sub and super groupings. 


\begin{tabular}{l|c|c|c}
\hline Order & $\begin{array}{c}\text { Number } \\
\text { of } \\
\text { Families }\end{array}$ & $\begin{array}{c}\text { Number } \\
\text { of Genera }\end{array}$ & $\begin{array}{c}\text { Number } \\
\text { of } \\
\text { Species }\end{array}$ \\
\hline Archaeognatha & 2 & 15 & 18 \\
\hline Zygentoma & 3 & 3 & 3 \\
\hline Odonata & 34 & 243 & 488 \\
\hline Ephemeroptera & 26 & 108 & 378 \\
\hline Zoraptera & 1 & 1 & 1 \\
\hline Dermaptera & 4 & 6 & 7 \\
\hline Plecoptera & 15 & 84 & 199 \\
\hline Orthoptera & 30 & 299 & 603 \\
\hline Mantophasmatodea & 1 & 1 & 1 \\
\hline Grylloblattodea & 1 & 1 & 0 \\
\hline Embioptera & 1 & 2 & 2 \\
\hline Phasmatodea & 6 & 20 & 28 \\
\hline Mantodea & 11 & 74 & 76 \\
\hline Blattodea & 8 & 82 & 95 \\
\hline Isoptera & 6 & 91 & 186 \\
\hline Thysanoptera & 3 & 14 & 30 \\
\hline Hemiptera & 101 & 1245 & 2730 \\
\hline Psocoptera & 12 & 17 & 19 \\
\hline Hymenoptera & 74 & 1500 & 4418 \\
\hline Raphidioptera & 2 & 9 & 11 \\
\hline Megaloptera & 2 & 10 & 22 \\
\hline Neuroptera & 14 & 65 & 153 \\
\hline Strepsiptera & 3 & 10 & 36 \\
\hline Coleoptera & 124 & 2287 & 7452 \\
\hline Trichoptera & 43 & 320 & 1269 \\
\hline Lepidoptera & 134 & 6554 & 21626 \\
\hline Siphonaptera & 6 & 14 & 20 \\
\hline Mecoptera & 4 & 5 & 10 \\
\hline Diptera & 118 & 1574 & 5460 \\
\hline & $\mathbf{7 8 9}$ & $\mathbf{1 4 6 5 4}$ & $\mathbf{4 5 3 4 1}$ \\
\hline & & & \\
\hline
\end{tabular}

1 


\section{Figure 1 (on next page)}

Data inventory throughout curation process.

A percent density histogram of the number of sequences per species $(A)$ shows the distribution of redundancy within the NCBI Nucleotide entries used. The dashed blue line and solid red line indicate the median and mean number of sequences per species, respectively. Inventories of the number of sequences $(A)$ and genera $(B)$ input into the curation process, following Metaxa2 extraction and following dereplication of redundant sequences and curation of taxonomic lineages. 
A

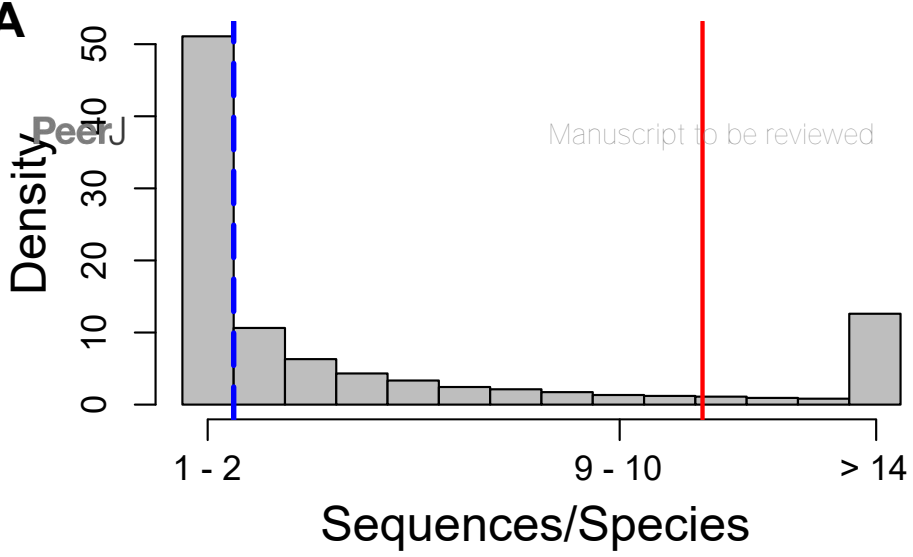

B

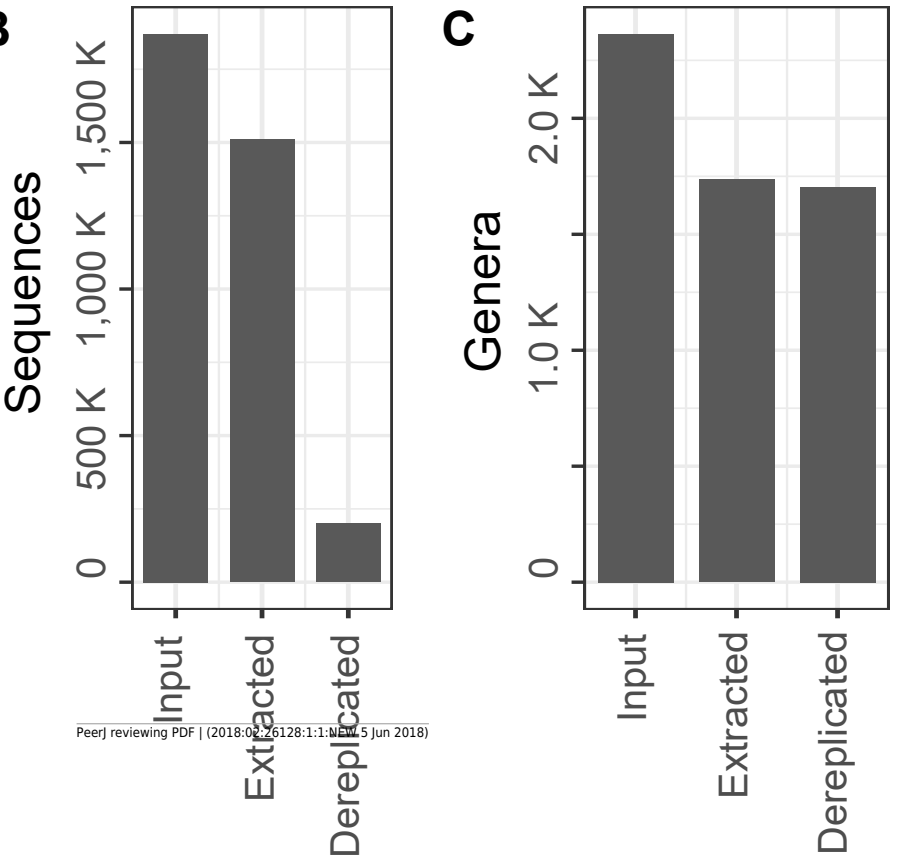




\section{Figure 2 (on next page)}

Estimating reliability score accuracy.

A logistic regression analysis of case-by-case classification accuracy, ' 1 ' indicating a falsepositive identification and ' 0 ' indicating a true-positive identification, regressed against classification reliability score for half length (A) and full length (B) test sequence cases. A best fit local polynomial regression line (solid blue with 95 percent confidence interval) was used to estimate the relationship between reliability score and the probability of misclassification. Dashed red lines illustrate the hypothetically ideal 1 to 1 relationship between error probability and the Metaxa2 reliability score, an estimate of classification confidence. Solid black lines highlight the 10 percent error probability. 
A

Peer.J

Half Length

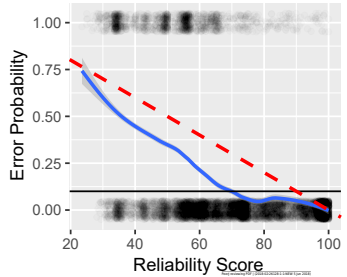

Full Length

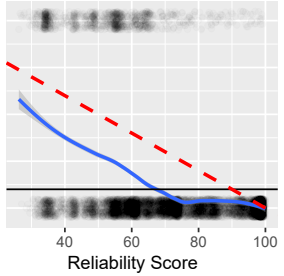


Figure 3 (on next page)

Classification performance accross entire testing corpus.

Mean proportion and standard error of true positives (TP), true negatives (TN), false negatives (FN) and false positives (FP) for the classification of all testing sequences, conducted on both the full-length and half-length sequences (A). Mean and standard error of the false discovery rate for both full length and half length sequences, as measured in errors per assignment, during classification of all testing sequences (B). 
Figure 4 (on next page)

Overclassification analysis.

Proportional species level overclassification rate (A) and genus level classification performance (B) for test sequence cases from species not represented in the corresponding training data. Proportional genus level overclassification rate (C), and family level classification performance (D) for test sequence cases from genera not represented in the corresponding training data. 


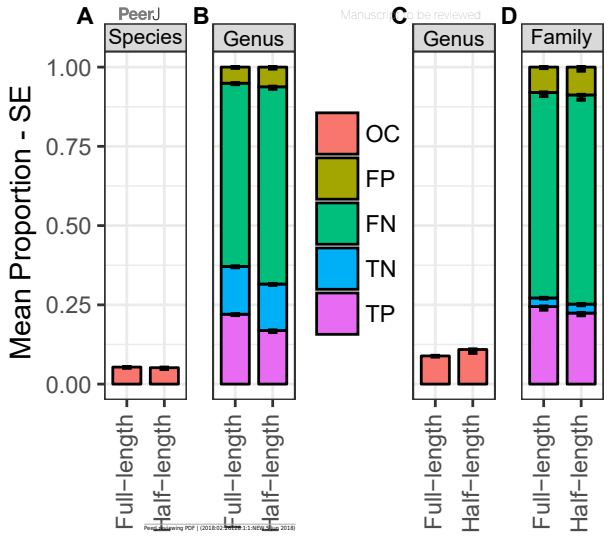


Figure $\mathbf{5}$ (on next page)

Classification performance by order.

Mean and standard error of the proportion of sequences assigned and false discovery rate, as measured in errors per assignment, measured at the family, genus and species levels for sequences belonging to each order. These results represent classification performance by arthropod order for the full length sequences only and orders with fewer than 100 test sequence cases at any of the ranks analyzed were excluded from analysis. 
